Research Article

\title{
The Existence of Fixed Points for a Different Type of Contractions on Partial $b$-Metric Spaces
}

\author{
Muhammad Nazam (D), ${ }^{1}$ Hüseyin Işik $\left(\mathbb{D},{ }^{2}\right.$ Khalil Javed $\left(D,{ }^{3}\right.$ Muhammad Naeem, ${ }^{3}$ \\ and Muhammad Arshad $\mathbb{i D}^{3}$ \\ ${ }^{1}$ Department of Mathematics, Allama Iqbal Open University H-8, Islamabad, Pakistan \\ ${ }^{2}$ Department of Engineering Science, Bandırma Onyedi Eylül University, Bandırma 10200, Balıkesir, Turkey \\ ${ }^{3}$ Department of Mathematics and Statistic, International Islamic University, Islamabad, Pakistan
}

Correspondence should be addressed to Hüseyin Işik; isikhuseyin76@gmail.com

Received 10 April 2021; Accepted 9 July 2021; Published 14 August 2021

Academic Editor: Ching-Feng Wen

Copyright (c) 2021 Muhammad Nazam et al. This is an open access article distributed under the Creative Commons Attribution License, which permits unrestricted use, distribution, and reproduction in any medium, provided the original work is properly cited.

\begin{abstract}
The aim of this study is to present fixed point results in the setting of partial $b$-metric spaces. A different type of contractions is used to prove fixed point results in the given space, which are real generalization of many well-known results. The readers are also provided with some very interesting examples to illustrate the feasibility of the proposed work.
\end{abstract}

\section{Introduction and Preliminaries}

The notion of metric space was initiated in 1906 by French Mathematician Frechet [1]. In metric fixed point theory, the Banach contraction principle [2] is one of the most fundamental tools to investigate the existence and uniqueness of solutions for contraction maps in a complete metric space. Since the inception of this principle, many authors studied fixed point theory vividly and enriched this field with different ideas. This classical result was generalized in different spaces, and different structures were attained using this topic and one may recall the existing notions, partial $b$-metric spaces [3], $\Re$-partial $b$-metric spaces [4], fuzzy cone $b$-metric spaces [5], $G_{b}$-metric spaces [6], orthogonal partial $b$-metric spaces [7], orthogonal $m$-metric spaces [8], and several others. More details can be found in [9-13].

One important and extensively used generalization of metric space is the notion of $b$-metric spaces, which was introduced by Czerwik [14] and is defined as follows.

Definition 1 (see [14]). Let $\Im$ be a nonempty set and $s \geq 1$ be a given real number. A function $b: \mathfrak{I} \times \mathfrak{I} \longrightarrow[0, \infty)$ is referred as a $b$-metric if the following conditions hold for all $\sigma, v, \varsigma \in \mathfrak{\Im}:$

$$
\begin{aligned}
& \left(b_{i}\right) b(\sigma, \varsigma)=0 \Leftrightarrow \sigma=\varsigma \\
& \left(b_{i i}\right) b(\sigma, \varsigma)=b(\varsigma, \sigma) \\
& \left(b_{i i i}\right) b(\sigma, \varsigma) \leq s[b(\sigma, v)+b(v, \varsigma)]
\end{aligned}
$$

The triplet $(\mathfrak{I}, b, s)$ is called a $b$-metric space.

Example 1 (see [15]). Let $\mathfrak{\Im}=\{1,2,3, \ldots\} \cup \infty$ and define $b: \mathfrak{I} \times \mathfrak{I} \longrightarrow[0, \infty)$ by

$$
b(\sigma, \varsigma)= \begin{cases}\left|\frac{1}{\sigma}-\frac{1}{\varsigma}\right|, & \text { if } \sigma, \varsigma \text { are even or } \sigma \varsigma=\infty, \\ 0, & \text { if } \sigma=\varsigma \\ 5, & \text { if } \rho, v \text { are odd or } \sigma \neq \varsigma, \\ 2, & \text { otherwise. }\end{cases}
$$

Then, $(\mathfrak{I}, b, s)$ is a $b$-metric space with $s=3$. 
Matthews [16], while working on networking, observed that a self-distance may not be zero, i.e., $\mathrm{d}(\sigma, \sigma) \neq 0$. A loop is a good example of this case. He not only generalized the classical Banach fixed point theorem but also established some convergence criterion in this setting to ensure existence of a fixed point. His further investigations led him to the introduction of partial metric space, which is defined as follows.

Definition 2 (see [16]). Let $\mathfrak{\Im}$ be a nonempty set. A mapping ב: $\mathfrak{I} \times \mathfrak{I} \longrightarrow[0, \infty)$ is referred as a partial metric if, for all $\sigma, \varsigma, v \in \mathfrak{I}$,

$$
\begin{aligned}
& \left.\left(\beth_{i}\right) \sigma=\varsigma \Leftrightarrow\right\rfloor(\sigma, \sigma)=\beth(\sigma, \varsigma)=\beth(\varsigma, \varsigma) \\
& \left(\beth_{i i}\right) \beth(\sigma, \sigma) \leq \beth(\sigma, \varsigma) \\
& \left(\beth_{i i i}\right) \beth(\sigma, \varsigma)=\beth(\varsigma, \sigma) \\
& \left(\beth_{i v}\right) \beth(\sigma, \varsigma) \leq \beth(\sigma, v)+\beth(v, \varsigma)-\beth(v, v)
\end{aligned}
$$

Then, pair $(\mathfrak{I}, \beth)$ is referred as a partial metric space.

Example 2 (see [16]). Let $\mathfrak{I}=[0, \infty)$ and define $\mathrm{J}: \mathfrak{J} \times \mathfrak{I}$ $\longrightarrow[0, \infty)$ by

$$
\beth(\sigma, \varsigma)=\max \{\sigma, \varsigma\} \quad \text { for all } \sigma, \varsigma \in \mathfrak{I} .
$$

Then, the pair $(\mathfrak{I}, \beth)$ is a partial metric space.

Example 3 (see [16]). Let $\mathfrak{I}=\{[\sigma, \varsigma]: \sigma, \varsigma \in(0, \infty)\}$ and define $\beth$ : $\mathfrak{I} \times \mathfrak{I} \longrightarrow[0, \infty)$ by

$$
\beth([\sigma, \varsigma],[v, \rho])=\max \{\varsigma, \rho\}-\min \{\sigma, v\} .
$$

The pair $(\mathfrak{I}, \beth)$ is a partial metric space.

Definition 3 (see [16]). Let $(\mathfrak{I}, \beth$ ) be a partial metric space and $\left\{\sigma_{\kappa}\right\}$ be a sequence in $\mathfrak{\Im}$. Then,

(i) $\left\{\sigma_{\kappa}\right\}$ converges in $\mathfrak{J} \Leftrightarrow \beth(\varsigma, \varsigma)=\lim _{\kappa \rightarrow \infty} \beth\left(\varsigma, \sigma_{\kappa}\right)$.

(ii) $\left\{\sigma_{\kappa}\right\}$ is referred as a Cauchy sequence if $\lim _{\kappa, j \rightarrow \infty} \beth\left(\sigma_{\kappa}, \sigma_{j}\right)$ exists and is finite.

(iii) A partial metric space is complete if each Cauchy sequence converges in $\mathfrak{J}$ satisfying

$$
J(\varsigma, \varsigma)=\lim _{\kappa, j \longrightarrow \infty} \beth\left(\sigma_{\kappa}, \sigma_{j}\right) .
$$

The concept of partial metric space was further extended to partial $b$-metric space by Shukla [3] in 2014 by combining the partial metric space and $b$-metric space.

Definition 4 (see [3]). Let $\mathfrak{\Im}$ be a nonempty set and $s \geq 1$ be a given real number. A mapping $\beth_{b}: \mathfrak{I} \times \mathfrak{I} \longrightarrow[0, \infty)$ is referred as a partial $b$-metric if, for all $\sigma, \varsigma, v \in \mathfrak{J}$,

$$
\begin{aligned}
& \left(\beth_{b_{i}}\right) \sigma=v \Leftrightarrow \beth_{b}(v, v)=\beth_{b}(v, \sigma)=\beth_{b}(\sigma, \sigma) \\
& \left(\beth_{b_{i i}}\right) \beth_{b}(v, v) \leq \beth_{b}(\sigma, v) \\
& \left(\beth_{b_{i i i}}\right) \beth_{b}(\varsigma, \sigma)=\beth_{b}(\sigma, \varsigma)
\end{aligned}
$$

$$
\left(\beth_{b_{i v}}\right) \beth_{b}(\sigma, \varsigma) \leq s\left[\beth_{b}(\sigma, v)+\beth_{b}(v, \varsigma)\right]-\beth_{b}(v, v)
$$

The triplet $\left(\mathfrak{I}, \beth_{b}, s\right)$ is referred as a partial $b$-metric space with $s \in[1, \infty)$.

Definition 5 (see [3]). Let $\left(\mathfrak{I}, \beth_{b}, s\right)$ be a partial $b$-metric space and $\left\{\sigma_{\kappa}\right\}$ be a sequence in $\mathfrak{\Im}$. Then,

(i) $\left\{\sigma_{\kappa}\right\}$ converges in $\mathfrak{\Im} \Leftrightarrow \beth_{b}(\varsigma, \varsigma)=\lim _{\kappa \longrightarrow \infty} \beth_{b}\left(\varsigma, \sigma_{\kappa}\right)$.

(ii) $\left\{\sigma_{n}\right\}$ is referred as a Cauchy sequence if $\lim _{\kappa, j \longrightarrow \infty} \beth_{b}\left(\sigma_{\kappa}, \sigma_{j}\right)$ exists and is finite.

(iii) A partial $b$-metric space is complete if each Cauchy sequence converges in $\mathfrak{I}$ such that

$$
\beth_{b}(\varsigma, \varsigma)=\lim _{\kappa, j \longrightarrow \infty} \beth_{b}\left(\sigma_{\kappa}, \sigma_{j}\right) .
$$

\section{Remark 1}

(i) In the partial $b$-metric space, $\beth_{b}(\sigma, \varsigma)=0 \Rightarrow \sigma=\varsigma$. The converse implication does not hold in general.

(ii) A partial $b$-metric is a generalization of a partial metric.

Example 4 (see [3]). Let $\mathfrak{\Im}=[0, \infty)($ with $\xi>1)$ and define $\beth_{b}: \mathfrak{I} \times \mathfrak{I} \longrightarrow[0, \infty)$ by

$$
\beth_{b}(\mathcal{\varkappa}, \varsigma)=\{\max (\varkappa, \varsigma)\}^{\xi}+(\mathcal{\varkappa}-\varsigma)^{\xi} \text { for all } \mathcal{x}, \varsigma \in \mathfrak{J} .
$$

$\beth_{b}$ is referred as a partial $b$-metric with $s=2^{\xi}$. For $\varsigma>0$, we have $\beth_{b}(\varsigma, \varsigma) \neq 0$, so $\beth_{b}$ is not a $b$-metric. On the contrary, for $\mathcal{x}, \sigma, \varsigma \in \mathfrak{I}$ with $\mathcal{x}>\sigma>\varsigma$, we have

$$
(\varkappa-\sigma)^{\xi}+(\sigma-\varsigma)^{\xi}<(\varkappa-\varsigma)^{\xi} .
$$

Observe that

$$
\begin{aligned}
I_{b}(\varkappa, \varsigma) & =\varkappa^{\xi}+(\sigma-\varsigma)^{\xi}, \\
I_{b}(\varkappa, \sigma)+\beth_{b}(\sigma, \varsigma)-\beth_{b}(\sigma, \sigma) & =\varkappa^{\xi}+(\varkappa-\sigma)^{\xi}+(\varkappa-\varsigma)^{\xi} .
\end{aligned}
$$

One can then write

$$
\beth_{b}(\varkappa, \sigma)+\beth_{b}(\sigma, \varsigma)-\beth_{b}(\sigma, \sigma)<\beth_{b}(\varkappa, \varsigma) .
$$

Thus, $\beth_{b}$ is not a partial metric.

Lemma 1 (see [3])

(1) In a partial b-metric space $\left(\mathfrak{I}, \beth_{b}, s\right),\left\{\sigma_{\kappa}\right\}$ is a $\mathrm{I}_{b}$-Cauchy sequence $\Leftrightarrow$ it is a b-Cauchy sequence in the b-metric space $(\mathfrak{I}, b)$.

(2) A partial b-metric space $\left(\mathfrak{I}, \beth_{b}\right)$ is $\beth_{b}$-complete $\Leftrightarrow$ the $b$-metric space $(\Im, b)$ is b-complete. Moreover, $\lim _{\kappa \longrightarrow \infty} b\left(\sigma, \sigma_{\kappa}\right)=0 \Leftrightarrow$ 


$$
\lim _{\kappa \longrightarrow \infty} \beth_{b}\left(\sigma, \sigma_{\kappa}\right)=\lim _{\kappa, \tau \longrightarrow \infty} \beth_{b}\left(\sigma_{\tau}, \sigma_{\kappa}\right)=\lim _{\kappa \longrightarrow \infty} \beth_{b}(\sigma, \sigma) .
$$

\section{Preliminary Results on Partial $b$-Metric Spaces}

Following [17], we state the following.

Definition 6. A function $F:(0, \infty) \longrightarrow(-\infty, \infty)$ belongs to $\Delta^{s}$ if

$\left(\mathrm{WF}^{1}\right) F$ is strictly increasing

$\left(\mathrm{WF}^{3}\right) \quad$ There exists $\xi \in(0,1)$ such that $\lim _{\sigma \longrightarrow \infty}(\sigma)^{\xi} F(\sigma)=0$

In the above definition, we omitted Wardowski's second condition, that is, $\left(\mathrm{WF}^{2}\right)$. For every sequence $\left\{\sigma_{\kappa}\right\} \subset(0, \infty)$,

$$
\lim _{\kappa \longrightarrow \infty} \sigma_{\kappa}=0 \Leftrightarrow \lim _{\kappa \longrightarrow \infty} F\left(\sigma_{\kappa}\right)=-\infty \text {. }
$$

Definition 7. Let $\left(\Im, \beth_{b}, s\right)$ be a partial $b$-metric space. A mapping $\psi: \mathfrak{J} \longrightarrow \mathfrak{J}$ is called an $F_{\beth_{b}}$-contraction if there are $F \in \Delta_{b}^{s}$ and $\tau>0$ so that

$$
\begin{aligned}
\sigma, \varsigma \in \mathfrak{I}, \beth_{b}(\psi(\sigma), \psi(\varsigma))>0 & \Rightarrow \tau+F\left(s \beth_{b}(\psi(\sigma), \psi(\varsigma))\right) \\
& \leq F\left(\beth_{b}(\sigma, \varsigma)\right) .
\end{aligned}
$$

Remark 2. In Example 5, there is an $F_{\beth_{b}}$-contraction mapping $\psi: \mathfrak{I} \longrightarrow \mathfrak{I}$, but it does not satisfy

$$
\beth_{b}(\psi(\sigma), \psi(\varsigma)) \leq p \beth_{b}(\sigma, \varsigma)
$$

for all $p \in[0,1)$.

Example 5. Let $\mathfrak{I}=\left\{\sigma_{\kappa}=2^{\kappa / 2} \kappa, \kappa \in \mathbb{N}\right\}$ and define $\beth_{b}$ : $\mathfrak{I} \times$ $\mathfrak{I}[0, \infty)$ by $\lrcorner_{b}(\sigma, \varsigma)=(\sigma \vee \varsigma)^{2}$. Then, the triplet $\left(\mathfrak{I}, \beth_{b}, s=2\right)$ is a partial $b$-metric space (with $s=2$ ). Consider $\psi: \mathfrak{I} \longrightarrow \mathfrak{I}$ as

$$
\psi(\sigma)= \begin{cases}\sigma_{k-1}=2^{(\kappa-1) / 2}(\kappa-1), & \text { if } \sigma=\sigma_{\kappa} \quad(\text { with } k=1,2, \ldots), \\ 0, & \text { if } \sigma=\sigma_{0}=0 .\end{cases}
$$

We claim that $\psi$ is an $F_{\beth_{b}}$-contraction in the partial $b$-metric space $\mathfrak{I}$ (with $\tau=1$ and $F(\omega)=\omega$, which is in $\Delta_{b}^{s}$ : $\left(\mathrm{WF}^{1}\right)$ and $\left(\mathrm{WF}^{3}\right)$ hold). For this, by this choice of $F$, we shall show that, for every $\sigma, \varsigma \in \mathfrak{I}$ so that $\psi(\sigma) \neq \psi(\varsigma)$, we have

$$
2 \beth_{b}(\psi(\sigma), \psi(\varsigma))-\beth_{b}(\sigma, \varsigma) \leq-\tau .
$$

For this, let $\sigma=\sigma_{\kappa+k}$ and $\sigma=\sigma_{\kappa}$. Then,

$$
\begin{aligned}
& 2 \beth_{b}\left(\psi\left(\sigma_{\kappa+k}\right), \psi\left(\sigma_{\kappa}\right)\right)-\beth_{b}\left(\sigma_{\kappa+k}, \sigma_{\kappa}\right) \\
& =2 \beth_{b}\left(2^{(\kappa+k-1) / 2}(\kappa+k-1), 2^{(\kappa-1) / 2}(\kappa-1)\right)-\beth_{b}\left(2^{(\kappa+k) / 2}(\kappa+k), 2^{\kappa / 2} \kappa\right) \\
& =\left(2^{(\kappa+k) / 2}(\kappa+k-1) \vee 2^{\kappa / 2}(\kappa-1)\right)^{2}-\left(2^{(\kappa+k) / 2}(\kappa+k) \vee 2^{\kappa / 2} \kappa\right)^{2} \\
& =2^{\kappa}\left(\left(2^{\kappa / 2}(\kappa+k-1) \vee(\kappa-1)\right)^{2}-\left(2^{\kappa / 2}(\kappa+k) \vee \kappa\right)^{2}\right) \\
& =2^{\kappa}\left(\left(2^{\kappa / 2}(\kappa+k-1)\right)^{2}-\left(2^{\kappa / 2}(\kappa+k)\right)^{2}\right. \\
& =2^{\kappa}\left(2^{k}(\kappa+k-1)^{2}-\left(2^{k}(\kappa+k)^{2}\right)\right) \\
& =-2^{\kappa+k} \leq-1 .
\end{aligned}
$$

On the contrary, $\psi$ is not a Banach contraction. Indeed, 


$$
\begin{aligned}
& \lim _{\kappa \longrightarrow \infty} \frac{2 \beth_{b}\left(\psi\left(\sigma_{\kappa}\right), \psi\left(\sigma_{0}\right)\right)}{\beth_{b}\left(\sigma_{\kappa}, \sigma_{0}\right)}=\lim _{\kappa \longrightarrow \infty} \frac{2\left(\sigma_{\kappa-1} \vee \sigma_{0}\right)^{2}}{\left(\sigma_{\kappa} \vee \sigma_{0}\right)^{2}} \\
& =\lim _{\kappa \longrightarrow \infty} \frac{2\left(2^{(\kappa-1) / 2}(\kappa-1) \vee 0\right)^{2}}{\left(2^{\kappa / 2} \kappa \vee 0\right)^{2}}=\lim _{\kappa \longrightarrow \infty} \frac{\left(2^{\kappa / 2}(\kappa-1) \vee 0\right)^{2}}{\left(2^{\kappa / 2} \kappa \vee 0\right)^{2}} \\
& =\lim _{\kappa \longrightarrow \infty} \frac{\left(2^{\kappa / 2}(\kappa-1)\right)^{2}}{\left(2^{\kappa / 2} \kappa\right)^{2}}=\lim _{n \longrightarrow \infty} \frac{(\kappa-1)^{2}}{\kappa^{2}} \\
& =\lim _{\kappa \longrightarrow \infty} \frac{\kappa^{2}+1-2 \kappa}{\kappa^{2}}=\lim _{\kappa \longrightarrow \infty} 1+\frac{1}{\kappa^{2}}-\frac{2}{\kappa}=1 .
\end{aligned}
$$

It shows that $\beth_{b}(\psi(\sigma), \psi(\varsigma)) \leq p \beth_{b}(\sigma, \varsigma) \quad$ (for all $p \in[0,1))$ does not hold.

In [11], the authors introduced the following assumption:

$$
\begin{aligned}
& \left(\mathrm{CH}^{4}\right) \text { for all } \kappa \in \mathbb{N}, \tau>0 \\
& \tau+F\left(s \sigma_{\kappa}\right) \leq F\left(\sigma_{\kappa-1}\right) \Rightarrow \tau+F\left(s^{\kappa} \sigma_{\kappa}\right) \leq F\left(s^{\kappa-1} \sigma_{\kappa-1}\right)
\end{aligned}
$$

Go back to Example 5. We proceed as follows. Let $\tau+F\left(s \sigma_{\kappa}\right) \leq F\left(\sigma_{\kappa-1}\right)$, that is, $\tau+s \sigma_{\kappa} \leq \sigma_{\kappa-1}$. One writes

$$
\begin{aligned}
F\left(s^{\kappa} \sigma_{\kappa}\right)+\tau & =s^{\kappa} \sigma_{\kappa}+\tau=s^{\kappa-1}\left(s \sigma_{\kappa}\right)+\tau \leq s^{\kappa-1}\left(\sigma_{\kappa-1}-\tau\right)+\tau \\
& =\tau+s^{\kappa-1} \sigma_{\kappa-1}-\tau s^{\kappa-1}=\tau\left(1-s^{\kappa-1}\right)+s^{\kappa-1} \sigma_{\kappa-1} \\
& \leq s^{\kappa-1} \sigma_{\kappa-1} \\
& =F\left(s^{\kappa-1} \sigma_{\kappa-1}\right) .
\end{aligned}
$$

Thus, $\left(\mathrm{CF}^{4}\right)$ holds.

Definition 8. Let $\left\{a_{n}\right\}$ be a sequence in $(0, \infty)$ and $\left\{b_{n}\right\}$ be a sequence in $[0, \infty)$. We call $\left\{b_{n}\right\} \in O\left(a_{n}\right)$ if there exists $C>0$ satisfying $b_{n} \leq C a_{n}$, for all $n \in \mathbb{N}$.

Lemma 2. Let $\left\{\sigma_{d}\right\}$ be a sequence in the partial b-metric space $\left(\mathfrak{\Im}, \beth_{b}, s\right)$. Assume that

$$
\beth_{b}\left(\sigma_{d}, \sigma_{d+1}\right) \in \cup\left\{O\left(d^{-\delta}\right): \quad \delta>1+\log _{2} s\right\} .
$$

Then, $\left\{\sigma_{d}\right\}$ is a Cauchy sequence.

Proof. By (19), there exist $\delta>1+\log _{2} s$ and $D>0$ satisfying

$$
\beth_{b}\left(\sigma_{d}, \sigma_{d+1}\right) \leq D d^{-\delta} \quad \forall d \in \mathbb{N}
$$

Note that $2 s<2^{\delta}$. Choose $\mu \in \mathbb{N}$ satisfying $2 s^{1+(1 / \mu)}<2^{\delta}$; then, we have

$$
\begin{aligned}
2^{\mu} s^{1+\mu} & <2^{\mu \delta} \\
& \Rightarrow 2^{\mu(1-\delta)} s^{1+\mu}<1 .
\end{aligned}
$$

Define a mapping $\psi: \mathbb{N} \longrightarrow \mathbb{N}$ by

$$
\psi(d)=2+\frac{2^{\mu d}-1}{2^{\mu}-1} .
$$

For $a, c, d \in \mathbb{N}$ satisfying $\psi(a) \leq c<d \leq \psi(a+1)$, we have

$$
\begin{aligned}
& \beth_{b}\left(\sigma_{c}, \sigma_{d}\right) \leq s^{\psi(d-c)} \sum_{j=c}^{d-1} \beth_{b}\left(\sigma_{j}, \sigma_{j+1}\right) \\
& \leq s^{\psi(\psi(a+1)-\psi(a))} \sum_{j=\psi(a)}^{\psi(a+1)-1} \jmath_{b}\left(\sigma_{j}, \sigma_{j+1}\right) \\
& =s^{\psi\left(2^{\mu a}\right)} \sum_{j=\psi(a)}^{\psi(a+1)-1} \beth_{b}\left(\sigma_{j}, \sigma_{j+1}\right) \\
& =s^{\mu a} \sum_{j=\psi(a)}^{\psi(a+1)-1} \beth_{b}\left(\sigma_{j}, \sigma_{j+1}\right) \\
& \leq D s^{\mu a} \sum_{j=\psi(a)}^{\psi(a+1)-1} j-\delta \\
& \leq D s^{\mu a} \int_{\psi(a)-1}^{\psi(a+1)-1} t^{-\delta} \mathrm{d} t \\
& \leq D s^{\mu a} \int_{(\psi(a)-2+1) /\left(2^{\mu}-1\right)}^{(\psi(a+1)-2+1) /\left(2^{\mu}-1\right)} t^{-\delta} \mathrm{d} t \\
& =\frac{D s^{\mu a}}{1-\delta}\left[t^{1-\delta}\right]_{(\psi(a)-2+1) /\left(2^{\mu}-1\right)}^{(\psi(a+1)-2+1) /\left(2^{\mu}-1\right)} \\
& =D s^{\mu a} \frac{1-2^{\mu(1-\delta)}}{\delta-1}\left(2^{\mu}-1\right)^{\delta-1} 2^{\mu a(1-\delta)} \\
& =M\left(s 2^{1-\delta}\right)^{\mu a} \text {, }
\end{aligned}
$$

where $M=\left(\left(D\left(1-2^{\mu(1-\delta)}\right)\left(2^{\mu}-1\right)^{\delta-1}\right) /(\delta-1)\right)>0$. Since

$$
\left(s 2^{1-\delta}\right)^{\mu}=\frac{s^{\mu+1} 2^{\mu(1-\delta)}}{s}<\frac{1}{s} \leq 1,
$$

we have

$$
\lim _{\mu \longrightarrow \infty} M\left(s 2^{1-\delta}\right)^{\mu a}=0 .
$$

For $a, l, c, d \in \mathbb{N}$ such that $a<l, \psi(a) \leq c<\psi(a+1)$ and $\psi(l)<d \leq \psi(l+1)$, then, from (21) and (23), 


$$
\begin{aligned}
& \beth_{b}\left(\sigma_{c}, \sigma_{d}\right) \leq s \beth_{b}\left(\sigma_{c}, \sigma_{\psi(a+1)}\right)+s \beth_{b}\left(\sigma_{\psi(a+1)}, \sigma_{d}\right)-\beth_{b}\left(\sigma_{\psi(a+1)}, \sigma_{\psi(a+1)}\right) \\
& \left.\leq s \beth_{b}\left(\sigma_{c}, \sigma_{\psi(a+1)}\right)+s[s\lrcorner_{b}\left(\sigma_{\psi(a+1)}, \sigma_{\psi(a+2)}\right)+s \beth_{b}\left(\sigma_{\psi(a+2)}, \sigma_{d}\right)\right] \\
& -s \beth_{b}\left(\sigma_{\psi(a+2)}, \sigma_{\psi(a+2)}\right)-\beth_{b}\left(\sigma_{\psi(a+1)}, \sigma_{\psi(a+1)}\right) \\
& =s \beth_{b}\left(\sigma_{c}, \sigma_{\psi(a+1)}\right)+s^{2} \beth_{b}\left(\sigma_{\psi(a+1)}, \sigma_{\psi(a+2)}\right)+s^{2} \beth_{b}\left(\sigma_{\psi(a+2)}, \sigma_{d}\right) \\
& \left.-s^{2} \beth_{b}\left(\sigma_{\psi(a+2)}, \sigma_{\psi(a+2)}\right)-\right\lrcorner_{b}\left(\sigma_{\psi(a+1)}, \sigma_{\psi(a+1)}\right) \\
& \leq s M\left(s 2^{1-\delta}\right)^{\mu a}+s^{2} M\left(s 2^{1-\delta}\right)^{\mu(a+1)}+s^{3} M\left(s 2^{1-\delta}\right)^{\mu(a+2)} \\
& +s^{3} \beth_{b}\left(\sigma_{\psi(a+3)}, \sigma_{d}\right)-s^{2} \beth_{b}\left(\sigma_{\psi(a+3)}, \sigma_{\psi(a+3)}\right)-s^{1} M\left(s 2^{1-\delta}\right)^{\mu(a+1)}-s^{0} M\left(s 2^{1-\delta}\right)^{\mu(a)} \\
& \leq \sum_{\kappa=0}^{2} s^{(\kappa+1)} M\left(s 2^{1-\delta}\right)^{\mu(a+\kappa)}+s^{3} \beth_{b}\left(\sigma_{\psi(a+3)}, \sigma_{d}\right) \\
& -\sum_{\kappa=0}^{1} s^{\kappa} M\left(s 2^{1-\delta}\right)^{\mu(a+\kappa)}-s^{2} \beth_{b}\left(\sigma_{\psi(a+3)}, \sigma_{\psi(a+3)}\right) \\
& \leq \ldots \leq \sum_{\kappa=0}^{l-a-1} s^{(\kappa+1)} M\left(s 2^{1-\delta}\right)^{\mu(a+\kappa)}+s^{l-a} \beth_{b}\left(\sigma_{\psi(l)}, \sigma_{d}\right) \\
& -\sum_{v}^{l-k-1} s^{\kappa} M\left(s 2^{1-\delta}\right)^{\mu(a+\kappa)}-s^{l-k} \beth_{b}\left(\sigma_{\psi(a+\kappa+1)}, \sigma_{\psi(a+\kappa+1)}\right) \\
& \leq \sum_{\kappa=0}^{l-a-1} s^{(\kappa+1)} M\left(s 2^{1-\delta}\right)^{\mu(a+\kappa)}+s^{l-a} M\left(s 2^{1-\delta}\right)^{\mu l} \\
& -\sum_{\kappa=0}^{l-a-1} s^{\kappa} M\left(s 2^{1-\delta}\right)^{\mu(a+\kappa)}-s^{l-a} M\left(s 2^{1-\delta}\right)^{\mu(a+\kappa)} \leq \sum_{\kappa=0}^{l-a} s^{(\kappa+1)} M\left(s 2^{1-\delta}\right)^{\mu(a+\kappa)} \\
& -\sum_{\kappa=0}^{l-a} s^{\kappa} M\left(s 2^{1-\delta}\right)^{\mu(a+\kappa)} \leq \sum_{\kappa=0}^{\infty} s^{(\kappa+1)} M\left(s 2^{1-\delta}\right)^{\mu(a+\kappa)}-\sum_{\kappa=0}^{\infty} s^{\kappa} M\left(s 2^{1-\delta}\right)^{\mu(a+\kappa)} \\
& =M\left(s 2^{1-\delta}\right)^{\mu(a+\kappa)}\left(\frac{s}{1-s^{\mu+1} 2^{\mu(1-\delta)}}-\frac{1}{1-s^{\mu+1} 2^{\mu(1-\delta)}}\right) .
\end{aligned}
$$

By (25), $\left\{\sigma_{d}\right\}$ is a Cauchy sequence.

Lemma 3 (see [9]). Let $\left\{\sigma_{n}\right\}$ be a decreasing sequence in $(0, \infty)$. Assume that there is a mapping $F:(0, \infty) \longrightarrow(-\infty, \infty)$, $\tau \in(0, \infty)$, and $k \in(0,1)$ satisfying $\left(W F^{3}\right)$ so that

$$
n \tau+F\left(s^{n} \sigma_{n}\right) \leq F\left(\sigma_{0}\right) \text {. }
$$

Then, $\left\{\sigma_{n}\right\} \in O\left(n^{1 / k}\right)$.

Generally, a partial $b$-metric is discontinuous. Following Lemma 3, this statement is justified.
Lemma 4. Consider the convergent sequence $\left\{\sigma_{\epsilon}\right\}$ in the partial b-metric space $\left(\mathfrak{I}, \beth_{b}, s\right)$. Suppose that $\lim _{\epsilon \longrightarrow \infty} \sigma_{\epsilon}=\sigma$ and $\sigma, \varsigma \in \mathfrak{\Im}$; then,

$$
\frac{1}{s} \beth_{b}(\sigma, \varsigma) \leq \liminf _{\varepsilon \longrightarrow \infty} \beth_{b}\left(\sigma_{\varepsilon}, \varsigma\right) \leq \limsup _{\varepsilon \longrightarrow \infty} \beth_{b}\left(\sigma_{\varepsilon}, \varsigma\right) \leq s \beth_{b}(\sigma, \varsigma) .
$$

Proof. Since $\left(\mathfrak{I}, \beth_{b}, s\right)$ is a partial $b$-metric space, by $\left(\beth_{b_{i v}}\right)$, we obtain 


$$
\begin{aligned}
\beth_{b}(\sigma, \varsigma) & \leq s\left[\beth_{b}\left(\sigma, \sigma_{\varepsilon}\right)+\beth_{b}\left(\sigma_{\varepsilon}, \varsigma\right)\right]-\beth_{b}\left(\sigma_{\varepsilon}, \sigma_{\varepsilon}\right) \\
& \leq s\left[\beth_{b}\left(\sigma, \sigma_{\varepsilon}\right)+\beth_{b}\left(\sigma_{\varepsilon}, \varsigma\right)\right] \\
& \Rightarrow \frac{1}{s} \beth_{b}(\sigma, \varsigma)-\beth_{b}\left(\sigma, \sigma_{\varepsilon}\right) \leq \beth_{b}\left(\sigma_{\varepsilon}, \varsigma\right) .
\end{aligned}
$$

Taking lim inf, we obtain

$$
\frac{1}{s} \beth_{b}(\sigma, \varsigma) \leq \liminf _{\varepsilon} \beth_{b}\left(\sigma_{\mathcal{\varepsilon}}, \varsigma\right) \text {. }
$$

Again, by $\left(\beth_{b_{i v}}\right)$, we obtain

$$
\begin{aligned}
\beth_{b}\left(\sigma_{\varepsilon}, \varsigma\right) & \left.\leq s\left[\beth_{b}\left(\sigma_{\varepsilon}, \sigma\right)+\beth_{b}(\sigma, \varsigma)\right]-\right\lrcorner_{b}(\sigma, \sigma) \\
& \leq s\left[\beth_{b}\left(\sigma_{\varepsilon}, \sigma\right)+\beth_{b}(\sigma, \varsigma)\right] \\
& \left.\Rightarrow \beth_{b}\left(\sigma_{\varepsilon}, \varsigma\right) \leq s\right\lrcorner_{b}\left(\sigma_{\varepsilon}, \sigma\right)+s \beth_{b}(\sigma, \varsigma) .
\end{aligned}
$$

Taking lim sup,

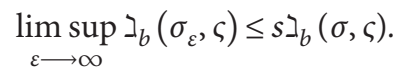

Also,

$$
\liminf _{\varepsilon \longrightarrow \infty} \beth_{b}\left(\sigma_{\varepsilon}, \varsigma\right) \leq \limsup _{\varepsilon \longrightarrow \infty} \beth_{b}\left(\sigma_{\varepsilon}, \varsigma\right) .
$$

Combining (30), (32), and (33), we have

$$
\frac{1}{s} \beth_{b}(\sigma, \varsigma) \leq \liminf _{\varepsilon \longrightarrow \infty} \beth_{b}\left(\sigma_{\varepsilon}, \varsigma\right) \leq \limsup _{\varepsilon \longrightarrow \infty} \beth_{b}(\sigma \varepsilon, \varsigma) \leq s \beth_{b}(\sigma, \varsigma) .
$$

\section{On $(\gamma, F)$-Weak Contractions}

We shall investigate the existence of a fixed point for $(\gamma, F)$-weak contraction mappings in partial $b$-metric spaces. We shall also provide some examples in support of main results.

Definition 9. Let $\psi: \mathfrak{I} \longrightarrow \mathfrak{J}$ and $\alpha_{s}: \mathfrak{I} \times \mathfrak{I} \longrightarrow[0, \infty)$ be two mappings and $\left(\mathfrak{I}, \beth_{b}, s \geq 1\right)$ be a partial $b$-metric space. Such $\psi$ is $\alpha_{s}$-admissible if

$$
\alpha_{s}(\sigma, \varsigma) \geq s^{2} \Rightarrow \alpha_{s}(\psi(\sigma), \psi(\varsigma)) \geq s^{2} \quad \text { for all } \sigma, \varsigma \in \mathfrak{I} .
$$

Example 6. Let $\mathfrak{I}=(0, \infty)$. Define $\psi: \mathfrak{I} \longrightarrow \mathfrak{I}$ and $\alpha_{s}: \mathfrak{I} \times$ $\mathfrak{\Im} \longrightarrow[0, \infty)$ by

$$
\begin{aligned}
\psi(\sigma) & =\ln \sigma, \quad \text { for all } \sigma \in \mathfrak{I}, \\
\alpha_{s}(\sigma, \varsigma) & = \begin{cases}2, & \text { if } \sigma \geq \varsigma, \\
0, & \text { if } \sigma<\varsigma .\end{cases}
\end{aligned}
$$

Then, $\psi$ is $\alpha_{s}$-admissible.

Definition 10. Let $\left(\mathfrak{I}, \beth_{b}, s\right)$ be a partial $b$-metric space and $\psi: \mathfrak{I} \longrightarrow \mathfrak{I}$ and $\alpha_{s}: \mathfrak{I} \times \mathfrak{I} \longrightarrow[0, \infty)$ be two mappings. Then, $\psi$ is triangular $\alpha_{s}$-admissible if

(1) $\alpha_{s}(\sigma, \varsigma) \geq s^{2}$ implies $\alpha_{s}(\psi(\sigma), \psi(\varsigma)) \geq s^{2}$
(2) $\alpha_{s}(\sigma, \varsigma) \geq s^{2}, \alpha_{s}(\varsigma, v) \geq s^{2}$ implies $\alpha_{s}(\sigma, v) \geq s^{2}$, for all $\sigma, \varsigma, v \in \mathfrak{J}$

Example 7. Let $\mathfrak{\Im}=(0, \infty), \psi(\sigma)=\sqrt[3]{\sigma}$, and $\alpha_{s}(\sigma, \varsigma)=e^{\sigma-\varsigma}$. Here, $\psi$ is triangular $\alpha_{s}$-admissible. Indeed, if $\alpha_{s}(\sigma$, $\varsigma)=e^{\sigma-\varsigma} \geq s^{2}$, then $\sigma \geq \varsigma$, which implies $\psi(\sigma) \geq \psi(\varsigma)$. That is, $\alpha_{s}(\psi(\sigma), \psi(\varsigma))=e^{\psi(\sigma)-\psi(\varsigma)} \geq s^{2}$. Also, if $\alpha_{s}(\sigma, \varsigma) \geq s^{2}$ and $\alpha_{s}(\varsigma, v) \geq s^{2}$, then $\sigma-\varsigma \geq 0, \varsigma-v \geq 0$. That is, $\sigma-v \geq 0$, so $\alpha_{s}(\sigma, v)=e^{\sigma-v} \geq s^{2}$.

Remark 3. For the given $\alpha_{s}$, there is $\gamma: \mathfrak{I} \times \mathfrak{I} \longrightarrow[0, \infty)$ defined by $\gamma(\sigma, \varsigma)=\left(\alpha_{s}(\sigma, \varsigma) / s^{2}\right)$ having the following conditions:

(1) $\psi$ is $\alpha_{s}$-admissible iff $\psi$ is $\gamma$-admissible, i.e.,

$$
\gamma(\sigma, \varsigma) \geq 1 \Rightarrow \gamma(\psi(\sigma), \psi(\varsigma)) \geq 1, \quad \text { for all } \sigma, \varsigma \in \mathfrak{S} .
$$

(2) The partial $b$-metric space is $\alpha_{s}$-complete iff it is $\gamma$-complete.

Definition 11. Let $\left(\mathfrak{I}, \beth_{b}, s\right)$ be a partial $b$-metric space and $\psi, \phi: \mathfrak{I} \longrightarrow \mathfrak{I}$ and $\gamma: \mathfrak{I} \times \mathfrak{I} \longrightarrow[0, \infty)$ be three mappings. Then, the pair $(\psi, \phi)$ is weakly $\gamma$-admissible if

$$
\begin{aligned}
\gamma(\sigma, \varsigma) \geq 1 \Rightarrow \gamma(\psi(\sigma), \phi \psi(\sigma)) \geq 1, \\
\gamma(\phi(\varsigma), \psi \phi(\varsigma)) \geq 1, \quad \text { for all } \sigma, \varsigma \in \mathfrak{\Im} .
\end{aligned}
$$

Definition 12. Let $\left(\Im, \beth_{b}, s\right)$ be a partial $b$-metric space and $\psi, \phi: \mathfrak{I} \longrightarrow \mathfrak{I}$ and $\gamma: \mathfrak{I} \times \mathfrak{I} \longrightarrow[0, \infty)$ be three mappings. The pair $(\psi, \phi)$ is triangular weakly $\gamma$-admissible if

(1) $\gamma(\sigma, \varsigma) \geq 1 \Rightarrow \gamma(\psi(\sigma), \phi \psi(\sigma)) \geq 1 \quad$ and $\quad \gamma(\phi(\varsigma), \psi \phi$ $(\varsigma)) \geq 1$, for all $\sigma, \varsigma \in \mathfrak{J}$

(2) $\gamma(\sigma, \varsigma) \geq 1, \gamma(\varsigma, v) \geq 1$ implies $\gamma(\sigma, v) \geq 1$, for all $\sigma, \varsigma, v \in \mathfrak{J}$

Example 8. Let $\mathfrak{\Im}=[0, \infty)$ and define $\psi, \phi: \mathfrak{I} \longrightarrow \mathfrak{J}$ by

$$
\begin{aligned}
& \psi(\sigma)= \begin{cases}\sigma^{1 / 2} & \text { if } \sigma \in[0,1), \\
1, & \text { if } \sigma \in[1, \infty),\end{cases} \\
& \phi(\sigma)= \begin{cases}\sigma, & \text { if } \sigma \in[0,1), \\
1, & \text { if } \sigma \in[1, \infty) .\end{cases}
\end{aligned}
$$

Define $\gamma: \mathfrak{I} \times \mathfrak{I} \longrightarrow[0, \infty)$ by

$$
\gamma(\sigma, \varsigma)= \begin{cases}e^{\sigma-\varsigma}, & \text { if } \sigma, \varsigma \in[0,1), \\ 0, & \text { if } \sigma, \varsigma \in[1, \infty) .\end{cases}
$$

If $\gamma(\sigma, \varsigma) \geq 1$ and $\gamma(\varsigma, v) \geq 1$, then

$$
\begin{aligned}
\sigma-\varsigma & \geq 0, \\
\varsigma-v & \geq 0 \\
\Rightarrow \sigma-v & \geq 0 \\
\Rightarrow \gamma(\sigma, v) & \geq 1 .
\end{aligned}
$$


Next, if $\gamma(\sigma, \varsigma) \geq 1$, then

$$
\begin{aligned}
\gamma(\psi(\sigma), \phi \psi(\sigma)) & =\gamma\left(\sigma^{1 / 2}, \phi\left(\sigma^{1 / 2}\right)\right)=\gamma\left(\sigma^{1 / 2}, \sigma^{1 / 2}\right) \\
& =e^{\sigma^{1 / 2}-\sigma^{1 / 2}} \geq 1, \\
\gamma(\phi(\varsigma), \psi \phi(\varsigma)) & =\gamma(\varsigma, \psi(\varsigma))=\gamma\left(\varsigma, \varsigma^{1 / 2}\right)=e^{\varsigma-\varsigma^{1 / 2}} \geq 1 .
\end{aligned}
$$

Definition 13. Consider the partial $b$-metric space $\left(\mathfrak{I}, \beth_{b}, s\right)$ and two mappings $\gamma: \mathfrak{I} \times \mathfrak{I} \longrightarrow[0, \infty)$ and $\psi: \mathfrak{I} \longrightarrow \mathfrak{I}$. $\psi$ is $\gamma$-continuous if for given $v \in \mathfrak{I}$ and a sequence $\left\{\sigma_{\kappa}\right\}$ so that

$$
\begin{aligned}
\lim _{\kappa \longrightarrow \infty} \beth_{b}\left(\sigma_{\kappa}, v\right) & =0, \\
\gamma\left(\sigma_{\kappa}, \sigma_{\kappa+1}\right) & \geq 1 \\
\Rightarrow \lim _{\kappa \longrightarrow \infty} \beth_{b}\left(\psi\left(\sigma_{\kappa}\right), \psi(v)\right) & =0, \quad \text { for all } \kappa \in \mathbb{N} .
\end{aligned}
$$

Example 9. Let $\mathfrak{J}=[0, \infty)$ and define a partial $b$-metric $\beth_{b}: \mathfrak{I} \times \mathfrak{I} \longrightarrow[0, \infty)$ by

$$
\beth_{b}(\sigma, \varsigma)=(\sigma \vee \varsigma)^{2}, \quad \text { for all } \sigma, \varsigma \in \mathfrak{I} .
$$

Define $\gamma: \mathfrak{I} \times \mathfrak{I} \longrightarrow[0, \infty)$ and $\psi: \mathfrak{I} \longrightarrow \mathfrak{I}$ by

$$
\begin{aligned}
\gamma(\sigma, \varsigma) & = \begin{cases}\sigma^{2}+\varsigma^{2}+1, & \text { if } \sigma, \mu \in[0,1], \\
0, & \text { otherwise, }\end{cases} \\
\psi(\omega) & = \begin{cases}\sin (\pi \omega), & \text { if } \omega \in[0,1], \\
\cos (\pi \omega)+2, & \text { if } \omega \in(1, \infty) .\end{cases}
\end{aligned}
$$

$\psi$ is not continuous because

$$
\lim _{\omega \longrightarrow 1^{-}} \psi(\omega) \neq \lim _{\omega \longrightarrow 1^{+}} \psi(\omega)
$$

Next, we prove that $\psi$ is $\gamma$-continuous. For given $v=$ $0 \in[0, \infty)$ and $\sigma_{\kappa}=1 / \kappa$, we obtain

$$
\begin{aligned}
\lim _{\kappa \longrightarrow \infty} \beth_{b}\left(\sigma_{\kappa}, v\right) & =\lim _{\kappa \longrightarrow \infty} \beth_{b}\left(\frac{1}{\kappa}, 0\right)=\lim _{\kappa \longrightarrow \infty}\left(\frac{1}{\kappa} \vee 0\right)=0, \\
\gamma\left(\frac{1}{\kappa}, \frac{1}{\kappa+1}\right) & =\left(1+\left(\frac{1}{\kappa}\right)^{2}+\left(\frac{1}{\kappa+1}\right)^{2}\right) \geq 1 .
\end{aligned}
$$

Now,

$$
\begin{aligned}
\lim _{\kappa \longrightarrow \infty} \beth_{b}\left(\psi\left(\sigma_{\kappa}\right), \psi(v)\right) & =\lim _{\kappa \longrightarrow \infty} \beth_{b}\left(\psi\left(\frac{1}{\kappa}\right), \psi(0)\right) \\
& =\lim _{\kappa \longrightarrow \infty}\left(\sin \left(\frac{\pi}{\kappa}\right) \vee \sin (0)\right) \\
& =\lim _{\kappa \longrightarrow \infty}\left(\sin \left(\frac{\pi}{\kappa}\right)\right)=0 .
\end{aligned}
$$

Hence, $\psi$ is $\gamma$-continuous.

Definition 14. Let $\left(\Im, \beth_{b}, s\right)$ be a partial $b$-metric space and $\gamma$ be defined above. The partial $b$-metric space is $\gamma$-complete iff every Cauchy sequence $\left\{\sigma_{\kappa}\right\}$ in $\mathfrak{J}$ satisfying $\gamma\left(\sigma_{\kappa}, \sigma_{\kappa+1}\right) \geq 1$ is convergent to $\sigma \in \mathfrak{J}$, for all $\kappa \in \mathbb{N}$.

Remark 4. If $\left(\mathfrak{I}, \beth_{b}, s\right)$ is a complete partial $b$-metric space, then $\left(\mathfrak{J}, \beth_{b}, s\right)$ is a $\gamma$-complete partial $b$-metric space, but not conversely.

Example 10. Let $\mathfrak{\Im}=(0, \infty)$ and $\beth_{b}: \mathfrak{I} \times \mathfrak{I} \longrightarrow[0, \infty)$ be the partial $b$-metric defined by

$$
\beth_{b}(\sigma, \varsigma)=(\sigma \vee \varsigma)^{2}, \quad \text { for all } \sigma, \varsigma \in \mathfrak{I} .
$$

Define $\gamma: \mathfrak{I} \times \mathfrak{J} \longrightarrow[0, \infty)$ by

$$
\gamma(\sigma, \varsigma)= \begin{cases}e^{\beth_{b}(\sigma, \varsigma)}, & \text { if } \sigma, \varsigma \in[2,5] \\ 0, & \text { otherwise. }\end{cases}
$$

We can show that $\left(\mathfrak{I}, \beth_{b}, s\right)$ is not a complete partial $b$-metric space, but $\left(\mathfrak{I}, \beth_{b}, s\right)$ is a $\gamma$-complete partial $b$-metric space. Indeed, $\left\{\sigma_{\kappa}\right\}$ is a Cauchy sequence in $\mathfrak{I}$ satisfying $\gamma\left(\sigma_{\kappa}, \sigma_{\kappa+1}\right) \geq 1$ for all $\kappa \in \mathbb{N}$; then, $\sigma_{\kappa} \in[2,5]$. Since $[2,5]$ is a closed subset of $(-\infty, \infty)$, we can check that $\left([2,5], \beth_{b}, 2\right)$ is a complete partial $b$-metric space, and then, there is $v \in[2,5]$ such that $\sigma_{\kappa} \longrightarrow v$ as $\kappa \longrightarrow \infty$.

Definition 15. Let $\left(\mathfrak{J}, \beth_{b}, s\right)$ be a partial $b$-metric space and $\gamma: \mathfrak{I} \times \mathfrak{I} \longrightarrow[0, \infty)$ be a mapping. $\left(\mathfrak{I}, \beth_{b}, s\right)$ is called $\gamma$-regular if, for every sequence $\left\{\sigma_{\kappa}\right\} \subset \mathfrak{I}$ satisfying $\gamma\left(\sigma_{\kappa}, \sigma_{\kappa+1}\right) \geq 1$ and $\sigma_{\kappa} \longrightarrow v$ as $\kappa \longrightarrow \infty$, we have $\gamma\left(\sigma_{\kappa}, v\right) \geq 1$, for all $\kappa \in \mathbb{N}$.

Definition 16. Consider the partial $b$-metric space $\left(\Im, \beth_{b}, s\right)$. Then, the pair of self-mappings $(\psi, \phi)$ forms a $(\gamma, F)$-weak contraction if there exist $F \in \Delta_{b}^{s}$ and $\tau>0$ such that

$$
\begin{aligned}
\tau & +F\left(s \gamma(\sigma, \varsigma) \beth_{b}(\psi(\sigma), \phi(\varsigma))\right) \\
& \leq F\left(M_{1}(\sigma, \varsigma)\right), \quad \text { for all } \sigma, \varsigma \in \mathfrak{I},
\end{aligned}
$$

with $\gamma(\sigma, \varsigma) \geq 1$, whenever

$$
\min \left\{\gamma(\sigma, \varsigma) \beth_{b}(\psi(\sigma), \phi(\varsigma)), M_{1}(\sigma, \varsigma)\right\}>0,
$$

where

$$
M_{1}(\sigma, \varsigma)=\max \left\{\beth_{b}(\sigma, \varsigma), \beth_{b}(\sigma, \psi(\sigma)), \beth_{b}(\varsigma, \phi(\varsigma)), \frac{\beth_{b}(\sigma, \phi(\varsigma))+\beth_{b}(\varsigma, \psi(\sigma))}{2 s}\right\} .
$$

Theorem 1. Let the pair of self-mappings $(\psi, \phi)$ be a $(\gamma, F)$-weak contraction defined on a $\gamma$-complete partial $b$-metric space $\left(\mathfrak{J}, \beth_{b}, s\right)$. Let $k \in\left(0,\left(1 /\left(1+\log _{2} s\right)\right)\right)$. Suppose that 
(1) The pair $(\psi, \phi)$ is weakly $\gamma$-admissible.

(2) There exists $\sigma_{0} \in \mathfrak{I}$ such that $\gamma\left(\sigma_{0}, \psi\left(\sigma_{0}\right)\right) \geq 1$.

(3) (a) Either $\mathfrak{J}$ is $\gamma$-regular and $F$ is continuous.

(b) One of $\psi, \phi$ is $\gamma$-continuous.

Then, we have a sequence $\left\{\sigma_{\kappa}\right\}$ in $\mathfrak{I}$ such that $\sigma_{\kappa} \longrightarrow v \in \mathfrak{I}$. If $\gamma(v, v) \geq 1$, then $v$ is a common fixed point of the pair $(\psi, \phi)$. Moreover, if $\omega$ is another common fixed point of the pair $(\psi, \phi)$ satisfying $\gamma(v, \omega) \geq 1$, then $v=\omega$.

Proof. For $\sigma_{1}, \sigma_{2} \in \mathfrak{I}$ such that $\sigma_{1} \neq \sigma_{2}$, then $M_{1}\left(\sigma_{1}, \sigma_{2}\right)>0$. Let $\sigma_{0} \in \mathfrak{I}$ be as in (2). Consider the iterative sequence $\left\{\sigma_{\kappa}\right\}$ in $\mathfrak{J}$ such that $\sigma_{1}=\psi\left(\sigma_{0}\right)$ and $\sigma_{2}=\phi\left(\sigma_{1}\right)$, and generally, $\sigma_{2 \kappa+1}=\psi\left(\sigma_{2 \kappa}\right), \sigma_{2 \kappa}=\phi\left(\sigma_{2 \kappa-1}\right)$, for all $\kappa \in \mathbb{N} \cup\{0\}$.

Using the weakly $\gamma$-admissibility, we have

$$
\begin{aligned}
& \gamma\left(\psi\left(\sigma_{0}\right), \phi \psi\left(\sigma_{0}\right)\right)=\gamma\left(\sigma_{1}, \sigma_{2}\right) \geq 1, \\
& \gamma\left(\psi\left(\sigma_{1}\right), \phi \psi\left(\sigma_{1}\right)\right)=\gamma\left(\sigma_{2}, \sigma_{3}\right) \geq 1, \\
& \gamma\left(\psi\left(\sigma_{2}\right), \phi \psi\left(\sigma_{2}\right)\right)=\gamma\left(\sigma_{3}, \sigma_{4}\right) \geq 1, \\
& \gamma\left(\psi\left(\sigma_{3}\right), \phi \psi\left(\sigma_{3}\right)\right)=\gamma\left(\sigma_{4}, \sigma_{5}\right) \geq 1 .
\end{aligned}
$$

Continuing in this way,

$$
\begin{aligned}
\gamma\left(\psi\left(\sigma_{2 \kappa}\right), \phi \psi\left(\sigma_{2 \kappa}\right)\right) & =\gamma\left(\sigma_{2 \kappa+1}, \sigma_{2 \kappa+2}\right) \geq 1, \\
\gamma\left(\psi\left(\sigma_{2 \kappa-1}\right), \phi \psi\left(\sigma_{2 \kappa-1}\right)\right) & =\gamma\left(\sigma_{2 \kappa}, \sigma_{2 \kappa+1}\right) \geq 1 .
\end{aligned}
$$

Hence, $\quad \gamma\left(\sigma_{\kappa}, \sigma_{\kappa+1}\right) \geq 1$, for all $\kappa \in \mathbb{N} \cup\{0\}$. If $\beth_{b}\left(\psi\left(\sigma_{2 \kappa}\right), \phi\left(\sigma_{2 \kappa+1}\right)\right)=0$, then $\sigma_{2 \kappa}$ is a common fixed point of $\psi, \phi$. Let $\beth_{b}\left(\psi\left(\sigma_{2 \kappa}\right), \phi\left(\sigma_{2 \kappa+1}\right)\right)>0$. From the contractive condition (51),

$$
\begin{aligned}
\left.F(s\lrcorner_{b}\left(\sigma_{2 \kappa}, \sigma_{2 \kappa+1}\right)\right) & \leq F\left(s \gamma\left(\sigma_{2 \kappa}, \sigma_{2 \kappa+1}\right) \beth_{b}\left(\psi\left(\sigma_{2 \kappa}\right), \phi\left(\sigma_{2 \kappa+1}\right)\right)\right) \\
& \leq F\left(M_{1}\left(\sigma_{2 \kappa}, \sigma_{2 \kappa+1}\right)\right)-\tau \quad \forall \kappa \in \mathbb{N} \cup\{0\},
\end{aligned}
$$

where

$$
\begin{aligned}
M_{1}\left(\sigma_{2 \kappa}, \sigma_{2 \kappa+1}\right) & =\max \left\{\beth_{b}\left(\sigma_{2 \kappa}, \sigma_{2 \kappa+1}\right), \beth_{b}\left(\sigma_{2 \kappa+1}, \phi\left(\sigma_{2 \kappa}\right)\right), \beth_{b}\left(\sigma_{2 \kappa+1}, \phi\left(\sigma_{2 \kappa+1}\right)\right), \frac{\beth_{b}\left(\sigma_{2 \kappa}, \phi\left(\sigma_{2 \kappa+1}\right)\right)+\beth_{b}\left(\sigma_{2 \kappa+1}, \psi\left(\sigma_{2 \kappa}\right)\right)}{2 s}\right\} \\
& \left.=\max \left\{\beth_{b}\left(\sigma_{2 \kappa}, \sigma_{2 \kappa+1}\right),\right\lrcorner_{b}\left(\sigma_{2 \kappa}, \sigma_{2 \kappa+1}\right), \beth_{b}\left(\sigma_{2 \kappa+1}, \sigma_{2 \kappa+2}\right), \frac{\beth_{b}\left(\sigma_{2 \kappa}, \sigma_{2 \kappa+2}\right)+\beth_{b}\left(\sigma_{2 \kappa+1}, \sigma_{2 \kappa+1}\right)}{2 s}\right\} \\
& \leq \max \left\{\beth_{b}\left(\sigma_{2 \kappa}, \sigma_{2 \kappa+1}\right), \beth_{b}\left(\sigma_{2 \kappa+1}, \sigma_{2 \kappa+2}\right)\right\} .
\end{aligned}
$$

If $M_{1}\left(\sigma_{2 \kappa}, \sigma_{2 \kappa+1}\right)=\beth_{b}\left(\sigma_{2 \kappa+1}, \sigma_{2 \kappa+2}\right)$, then

$$
F\left(s \beth_{b}\left(\sigma_{2 \kappa+1}, \sigma_{2 \kappa+2}\right)\right) \leq F\left(\beth_{b}\left(\sigma_{2 \kappa+1}, \sigma_{2 \kappa+2}\right)\right)-\tau,
$$

which contradicts $\left(\mathrm{WF}^{1}\right)$. Therefore,

$$
F\left(s \beth_{b}\left(\sigma_{2 \kappa+1}, \sigma_{2 \kappa+2}\right)\right) \leq F\left(\beth_{b}\left(\sigma_{2 \kappa}, \sigma_{2 \kappa+1}\right)\right)-\tau, \quad \kappa \in \mathbb{N} \cup\{0\} .
$$

Similarly, we have

$F\left(s \beth_{b}\left(\sigma_{2 \kappa+2}, \sigma_{2 \kappa+3}\right)\right) \leq F\left(\beth_{b}\left(\sigma_{2 \kappa+1}, \sigma_{2 \kappa+2}\right)\right)-\tau, \quad \kappa \in \mathbb{N} \cup\{0\}$.

From (59) and (60), we have

$$
F\left(s \beth_{b}\left(\sigma_{\kappa}, \sigma_{\kappa+1}\right)\right) \leq F\left(\beth_{b}\left(\sigma_{\kappa-1}, \sigma_{\kappa}\right)\right)-\tau, \quad \kappa \in \mathbb{N} .
$$

Let $b_{\kappa}=\beth_{b}\left(\sigma_{\kappa}, \sigma_{\kappa+1}\right)$, for each $\kappa \in \mathbb{N} \cup\{0\}$; then, from (61) and $\left(\mathrm{CH}^{4}\right)$,

$$
\tau+F\left(s^{\kappa} b_{\kappa}\right) \leq F\left(s^{\kappa-1} b_{\kappa-1}\right), \quad \kappa \in \mathbb{N} .
$$

Continuing in this way, we obtain

$$
F\left(s^{\kappa} b_{\kappa}\right) \leq F\left(b_{0}\right)-\kappa \tau, \quad \kappa \in \mathbb{N} .
$$

So, $\quad\left\{b_{\kappa}\right\} \in O\left(\kappa^{-1 / k}\right)$ by Lemma 3. Since $(1 / k) \in\left(1+\log _{2} s, \infty\right)$, by Lemma $2,\left\{\sigma_{\kappa}\right\}$ is a Cauchy sequence. We know that $\mathfrak{I}$ is a $\gamma$-complete partial $b$-metric space; then, there is $v \in \mathfrak{J}$ satisfying $\sigma_{2 \kappa+1} \longrightarrow v$ and $\sigma_{2 \kappa+2} \longrightarrow v$ as $\kappa \longrightarrow \infty$. Using $\gamma$-continuity of $\psi$, one writes

$$
\begin{aligned}
v & =\lim _{\kappa \longrightarrow \infty} \sigma_{\kappa}=\lim _{\kappa \longrightarrow \infty} \sigma_{2 \kappa+1}=\lim _{\kappa \longrightarrow \infty} \sigma_{2 \kappa+2}=\lim _{\kappa \longrightarrow \infty} \psi\left(\sigma_{2 \kappa+1}\right) \\
& =\psi\left(\lim _{\kappa \longrightarrow \infty} \sigma_{2 \kappa+1}\right)=\psi(v) .
\end{aligned}
$$

If $\beth_{b}(v, \phi(v))>0$ as $\gamma(v, v) \geq 1$, then by the contractive condition (51), we have

$$
\begin{aligned}
\tau+F\left(s \beth_{b}(v, \phi(v))\right) & \leq \tau+F\left(s \gamma(v, v) \beth_{b}(\psi(v), \phi(v))\right) \\
& \leq F M_{1}(v, v)=F\left(\beth_{b}(v, \phi(v))\right) .
\end{aligned}
$$

It is a contradiction. Hence,

$$
\begin{aligned}
\beth_{b}(v, \phi(v)) & =0 \\
& \Rightarrow v=\phi(v) .
\end{aligned}
$$


Thus, we have $\psi(v)=\phi(v)=v$. Hence, $(\psi, \phi)$ has a common fixed point $v$.

If $\mathfrak{I}$ is $\gamma$-regular and $F$ is continuous, then there are two different cases. Firstly, if there is a subsequence $\left\{\sigma_{n_{\kappa}}\right\}_{\kappa \in \mathbb{N}} \subset\left\{\sigma_{n}\right\}_{n \in \mathbb{N}}$ satisfying

$$
\sigma_{n_{\kappa}}= \begin{cases}\psi(v), & \text { if } \kappa \in 2 n, \\ \phi(v), & \text { if } \kappa \in 2 n+1 .\end{cases}
$$

Then,

$$
\begin{aligned}
& v=\lim _{\kappa \longrightarrow \infty} \sigma_{n_{\kappa}}=\lim _{\kappa \longrightarrow \infty} \psi(v)=\psi(v), \\
& v=\lim _{\kappa \longrightarrow \infty} \sigma_{n_{\kappa}}=\lim _{\kappa \longrightarrow \infty} \phi(v)=\phi(v) .
\end{aligned}
$$

It is completed. Second, if there is no such subsequence of $\left\{\sigma_{\kappa}\right\}_{\kappa \in N}$, then there is $\mu_{0} \in \mathbb{N}$ such that, for each $\kappa \geq \mu_{0}$, we obtain

$$
\begin{aligned}
\beth_{b}\left(\psi\left(\sigma_{2 \kappa}\right), \phi(v)\right) & >0, \\
\beth_{b}\left(\phi\left(\sigma_{2 \kappa+1}\right), \psi(v)\right) & >0 .
\end{aligned}
$$

Since $\mathfrak{J}$ is $\gamma$-regular, one writes

$$
\begin{gathered}
\gamma\left(\sigma_{2 \kappa+1}, v\right) \geq 1, \\
\gamma\left(\sigma_{2 \kappa}, v\right) \geq 1 .
\end{gathered}
$$

By the contractive condition (51), we have

$$
\begin{aligned}
& \tau+F\left(s \gamma\left(\sigma_{2 \kappa}, v\right) \beth_{b}\left(\psi\left(\sigma_{2 \kappa}\right), \phi(v)\right)\right) \\
& \leq F \max \left\{\beth_{b}\left(\sigma_{2 \kappa}, v\right), \beth_{b}\left(\sigma_{2 \kappa}, \psi\left(\sigma_{2 \kappa}\right)\right), \beth_{b}(v, \phi(v)), \frac{\beth_{b}\left(\sigma_{2 \kappa}, \phi(v)\right)+\beth_{b}\left(v, \psi\left(\sigma_{2 \kappa}\right)\right)}{2 s}\right\} .
\end{aligned}
$$

Now, we prove that $\beth_{b}(v, \phi(v))=0$. Suppose, on the contrary, that $I_{b}(v, \phi(v))=q>0$. Put $\lambda_{\kappa}=I_{b}$ $\left(\sigma_{\kappa}, v\right)=0 \forall \kappa \in \mathbb{N}$. Since $\lim _{\kappa \rightarrow \infty} \sigma_{\kappa}=v$, there exists $\mu_{1} \in \mathbb{N}$ satisfying for each $\kappa \geq \mu_{1}$, both $\lambda_{\kappa}<(q / 2)$ and $b_{\kappa}<(q / 2)$ hold. Consequently, by (71), we obtain

$$
\begin{aligned}
\tau+ & \left.F\left(s \gamma\left(\sigma_{2 \kappa}, v\right)\right\lrcorner_{b}\left(\psi\left(\sigma_{2 \kappa}\right), \phi(v)\right)\right) \\
& \leq F\left(\max \left\{\lambda_{2 \kappa}, b_{2 \kappa}, q, \frac{\lrcorner_{b}\left(\sigma_{2 \kappa}, \phi(v)\right)+\lambda_{2 \kappa+1}}{2 s}\right\}\right) \\
& \leq F\left(\max \left\{\lambda_{2 \kappa}, b_{2 \kappa}, q, \frac{s \lambda_{2 \kappa}+s q+\lambda_{2 \kappa+1}}{2 s}\right\}\right) \\
& \leq F\left(\max \left\{\frac{q}{2}, \frac{q}{2}, q, \frac{(s q / 2)+s q+(q / 2)}{2 s}\right\}\right)=F(q) .
\end{aligned}
$$

Then, for every $\kappa \geq \max \left\{\mu_{0}, \mu_{1}\right\}$, we obtain

$$
\tau+F\left(s \gamma\left(\sigma_{2 \kappa}, v\right) \beth_{b}\left(\psi\left(\sigma_{2 \kappa}\right), \phi(v)\right)\right) \leq F\left(\beth_{b}(v, \phi(v))\right) \text {. }
$$

Since $F$ is increasing and continuous, by Lemma 4 and (73), we have

$$
\begin{aligned}
\tau+F\left(\beth_{b}(v, \phi(v))\right) \leq & \tau+F\left(s \gamma\left(\sigma_{2 \kappa}, v\right) \liminf _{\kappa \longrightarrow \infty} \beth_{b}\right. \\
& \left.\cdot\left(\psi\left(\sigma_{2 \kappa}\right), \phi(v)\right)\right) \\
\leq & \tau+\liminf _{\kappa \longrightarrow \infty} F\left(s \gamma\left(\sigma_{2 \kappa}, v\right) \beth_{b}\left(\psi\left(\sigma_{2 \kappa}\right), \phi(v)\right)\right) \\
\leq & F\left(\beth_{b}(v, \phi(v))\right) .
\end{aligned}
$$

The above result shows that $\tau \leq 0$, which is a contradiction. Then, $\beth_{b}(v, \phi(v))=0$, and hence, $v=\phi(v)$. Similarly, we can show that $v=\psi(v)$, and consequently, $v$ is a common fixed point of $\psi$ and $\phi$.

Now, we prove that self-mappings $\psi, \phi$ have a unique common fixed point. Let $v$ and $\omega$ be two common fixed points of $\psi$ and $\phi$. Then, $\psi(v)=v \neq \omega=\phi(\omega)$. It follows that

$$
\beth_{b}(\psi(v), \phi(\omega))=\beth_{b}(v, \omega)>0 .
$$

Since $\gamma(v, \omega) \geq 1$, by contractive condition, one writes

$$
\begin{aligned}
\tau+ & F\left(s \gamma(v, \omega) \beth_{b}(\psi(v), \phi(\omega))\right) \\
& \left.\leq F\left(\max \left\{\beth_{b}(v, \omega), \beth_{b}(\psi(v), v),\right\lrcorner_{b}(\phi(\omega), \omega), \frac{\left.\beth_{b}(\phi(\omega), v)+\right\lrcorner_{b}(\psi(v), \omega)}{2 s}\right\}\right) \\
= & F\left(\beth_{b}(v, \omega)\right) \leq F\left(s \gamma(v, \omega) \beth_{b}(v, \omega)\right) .
\end{aligned}
$$

It shows that $\tau \leq 0$, which is a contradiction. Hence, $\psi$ and $\phi$ have a unique common fixed point.
Example 11. Let $\mathfrak{I}=[0, \infty)$ and define $\beth_{b}, \gamma: \mathfrak{I} \times \mathfrak{I} \longrightarrow$ $[0, \infty)$ by 


$$
\begin{aligned}
\beth_{b}(\sigma, \varsigma) & =(\sigma \vee \varsigma)^{2}, \\
\gamma(\sigma, \varsigma) & = \begin{cases}K \geq 1, & \text { if } \sigma \geq \varsigma, \\
0, & \text { if } \sigma<\varsigma .\end{cases}
\end{aligned}
$$

So, $\mathfrak{I}$ is a $\gamma$-complete partial $b$-metric space with $s=2$.

Take $\psi, \phi: \mathfrak{I} \longrightarrow \mathfrak{I}$ as

$$
\begin{aligned}
& \psi(\sigma)=\ln \left(1+\frac{\sigma}{6}\right), \\
& \phi(\sigma)=\ln \left(1+\frac{\sigma}{7}\right) .
\end{aligned}
$$

Definitely, $\psi$ and $\phi$ are $\gamma$-continuous. To prove that pair $(\psi, \phi)$ is weakly $\gamma$-admissible, let $\sigma, \varsigma \in \mathfrak{J}$ be such that $\varsigma=\psi(\sigma)$, so $\varsigma=\ln (1+(\sigma / 6))$. We have

$$
\begin{aligned}
\psi(\sigma) & =\varsigma=\ln \left(\frac{\sigma}{6}+1\right) \geq \ln \left(1+\frac{\varsigma=\ln (1+(\sigma / 6))}{7}\right) \\
& =\ln \left(1+\frac{\varsigma}{7}\right)=\phi(\varsigma)=\phi \psi(\sigma) .
\end{aligned}
$$

Thus, $\gamma(\psi(\sigma), \phi \psi(\sigma)) \geq 1$. Now, let $v, \varsigma \in \mathfrak{J}$ be such that $v=\phi(\varsigma)$, so $v=\ln (1+(\varsigma / 7))$. Since

$$
\begin{aligned}
\phi(\varsigma) & =\ln \left(1+\frac{\varsigma}{7}\right) \geq \ln \left(1+\frac{\varsigma=\ln (1+(\varsigma / 7))}{6}\right) \\
& =\ln \left(1+\frac{v}{6}\right)=\psi(v)=\psi \phi(\varsigma),
\end{aligned}
$$

we get that $\gamma(\phi(\varsigma), \psi \phi(\varsigma)) \geq 1$. Hence, the pair $(\psi, \phi)$ is weakly $\gamma$-admissible.

Next, for all $\sigma, \varsigma \in \mathfrak{I}$ with $\sigma \geq \varsigma$, choose $\xi$ such that $(\xi / 2 K)>1+\log _{2} s$; then,

$$
\begin{aligned}
& 2 \gamma(\sigma, \varsigma) \beth_{b}(\psi(\sigma), \phi(\varsigma))=2 K(\psi(\sigma) \vee \phi(\varsigma))^{2} \\
& =2 K\left(\ln \left(\frac{\sigma}{6}+1\right) \vee \ln \left(1+\frac{\varsigma}{7}\right)\right)^{2}=2 K\left(\ln \left(1+\frac{\varsigma}{6}\right)\right)^{2} \\
& \leq 2 K\left(\ln \left(1+\frac{\sigma}{6}\right)\right)^{2} \leq \frac{2 K}{\xi} \beth_{b}(\sigma, \varsigma) \leq \frac{2 K}{\xi} M_{1}(\sigma, \varsigma) .
\end{aligned}
$$

The above result may be written as

$\ln \frac{\xi}{2 k}+\ln \left(2 \gamma(\sigma, \varsigma) \beth_{b}(\psi(\sigma), \phi(\varsigma))+1\right) \leq \ln \left(M_{1}(\sigma, \varsigma)+1\right)$.

Define $F:(0, \infty) \longrightarrow(-\infty, \infty)$ by

$$
F(v)=\ln (v+1), \quad \text { for all } v \in(0, \infty) .
$$

Then, $F \in \Delta_{b}^{s}$. Hence, for all $\sigma, \varsigma \in \mathfrak{J}$ such that $\beth_{b}(\psi(\sigma), \phi(\varsigma))>0$ and $\tau=\ln (\xi / 2 K)$, we obtain

$$
\tau+F\left(2 \gamma(\sigma, \varsigma) \beth_{b}(\psi(\sigma), \phi(\varsigma))\right) \leq F\left(M_{1}(\sigma, \varsigma)\right) .
$$

Consequently, the contractive condition (51) holds for all $\sigma, \varsigma \in \mathfrak{J}$. Hence, all the assumptions of Theorem 1 are verified. Thus, $\psi$ and $\phi$ have a unique common fixed point which is $v=0$.
Definition 17. Let $\left(\mathfrak{I}, \beth_{b}, s\right)$ be a partial $b$-metric space. Then, the self-mappings $\psi, \phi: \mathfrak{I} \longrightarrow \mathfrak{I}$ form a Hardy-Rogers $(\gamma, F)$-contraction if there exist $F \in \Delta_{b}^{s}$ and $\tau>0$ satisfying

$$
\tau+F\left(s \gamma(\sigma, \varsigma) \beth_{b}(\psi(\sigma), \phi(\varsigma))\right) \leq F(R(\sigma, \varsigma)), \quad \forall \sigma, \varsigma \in \mathfrak{J},
$$

with $\gamma(\sigma, \varsigma) \geq 1$, whenever

$$
\min \left\{\gamma(\sigma, \varsigma) \beth_{b}(\psi(\sigma), \phi(\varsigma)), R(\sigma, \varsigma)\right\}>0,
$$

where

$$
\begin{aligned}
R(\sigma, \varsigma)= & \xi_{1} \beth_{b}(\sigma, \varsigma)+\xi_{2} \beth_{b}(\sigma, \psi(\sigma))+\xi_{3} \beth_{b}(\varsigma, \phi(\varsigma)) \\
& +\xi_{4}\left[\beth_{b}(\sigma, \phi(\varsigma))+\beth_{b}(\psi(\sigma), \varsigma)\right],
\end{aligned}
$$

such that $\xi_{1}+\xi_{2}+\xi_{3}+2 s \xi_{4}=1$.

Theorem 2. Given the pair $(\psi, \phi): \mathfrak{I} \longrightarrow \mathfrak{\Im}$ of a Har$d y$-Rogers-type $(\gamma, F)$-contraction defined on a $\gamma$-complete partial b-metric space $\left(\Im, \beth_{b}, s\right)$. Consider $k \in(0$, $\left.\left(1 /\left(1+\log _{2} s\right)\right)\right)$. Suppose that

(1) The pair $(\psi, \phi)$ is weakly $\gamma$-admissible

(2) There exists $\sigma_{0} \in \mathfrak{I}$ such that $\gamma\left(\sigma_{0}, \psi\left(\sigma_{0}\right)\right) \geq 1$

(3) (a) $\mathfrak{I}$ is $\gamma$-regular and $F$ is continuous

(b) Either one of $\psi, \phi$ is $\gamma$-continuous

Then, we have a sequence $\left\{\sigma_{n}\right\}$ in $\mathfrak{I}$ such that $\sigma_{n} \longrightarrow v \in \mathfrak{I}$. If $\gamma(v, v) \geq 1$, then $v$ is a common fixed point of the pair $(\psi, \phi)$. Moreover, if $\omega$ is another fixed point of the pair $(\psi, \phi)$ satisfying $\gamma(v, w) \geq 1$, then $v=\omega$.

Proof. We have

$$
\begin{aligned}
R(\varsigma, \sigma)= & \xi_{1} \beth_{b}(\varsigma, \sigma)+\xi_{2} \beth_{b}(\psi(\sigma), \sigma)+\xi_{3} \beth_{b}(\phi(\varsigma), \varsigma) \\
& +\xi_{4}\left[\beth_{b}(\phi(\varsigma), \sigma)+\beth_{b}(\varsigma, \psi(\sigma))\right]=\xi_{1} \beth_{b}(\varsigma, \sigma) \\
& +\xi_{2} \beth_{b}(\psi(\sigma), \sigma) \\
& +\xi_{3} \beth_{b}(\phi(\varsigma), \varsigma)+2 s \xi_{4}\left[\frac{\beth_{b}(\sigma, \phi(\varsigma))+\beth_{b}(\varsigma, \phi(\sigma))}{2 s}\right] \\
\leq & \xi_{1} M_{1}(\sigma, \varsigma)+\xi_{2} M_{1}(\sigma, \varsigma)+\xi_{3} M_{1}(\sigma, \varsigma)+2 s \xi_{4} M_{1}(\sigma, \varsigma) \\
= & \left(\xi_{1}+\xi_{2}+\xi_{3}+2 s \xi_{4}\right) M_{1}(\sigma, \varsigma)=M_{1}(\sigma, \varsigma) .
\end{aligned}
$$

Inequality (85) implies (51), so the rest of proof follows from Theorem 1.

\section{Conclusion}

This paper contains a comparative study on partial $b$-metric spaces with examples. The obtained results generalize corresponding ones in the literature. We developed a methodology to obtain fixed point theorems in partial $b$-metric spaces. This paper may lead many researchers of this subject to investigate new fixed point theorems in partial $b$-metric spaces. 


\section{Data Availability}

The data used to support the findings of this study are available from the corresponding author upon request.

\section{Conflicts of Interest}

The authors declare that they have no conflicts of interest regarding the publication of this paper.

\section{Authors' Contributions}

All authors contributed equally and significantly in writing this article. All authors read and approved the final manuscript.

\section{References}

[1] M. M. Fréchet, "Sur quelques points du calcul fonctionnel," Rendiconti del Circolo Matematico di Palermo, vol. 22, no. 1, pp. 1-72, 1906.

[2] S. Banach, "Sur les opérations dans les ensembles abstraits et leur application aux équations intégrales," Fundamenta Mathematicae, vol. 3, pp. 133-181, 1922.

[3] S. Shukla, "Partial b-metric spaces and fixed point theorems," Mediterranean Journal of Mathematics, vol. 11, no. 2, pp. 703-711, 2014.

[4] M. U. Ali, Y. Guo, F. Uddin, H. Aydi, K. Javed, and Z. Ma, “On partial metric spaces and related fixed point results with applications," Journal of Function Spaces, vol. 2020, Article ID 6671828, 8 pages, 2020.

[5] S. S. Chauhan and V. Gupta, "Banach contraction theorem on fuzzy cone b-metric space," Journal of Applied Research and Technology, vol. 18, no. 4, pp. 154-160, 2020.

[6] V. Gupta, Ö. Ege, and R. Saini, "Some fixed point results in complete $G_{b}$-metric spaces," Dynamic Systems and Applications, vol. 30, no. 2, pp. 277-293, 2021.

[7] K. Javed, H. Aydi, F. Uddin, and M. Arshad, "On orthogonal partial metric spaces with an application," Journal of Mathematics, vol. 2021, Article ID 6692063, 7 pages, 2021.

[8] F. Uddin, C. Park, K. Javed, M. Arshad, and J. R. Lee, "Orthogonal $\mathrm{m}$-metric spaces and an application to solve integral equations," Advances in Difference Equations, vol. 2021, Article ID 159, 2021.

[9] M. Nazam, N. Hussain, A. Hussain, and M. Arshad, "Fixed point theorems for weakly $\beta$ - admissible pair of $F-$ contractions with application," Nonlinear Analysis: Modelling and Control, vol. 24, no. 6, pp. 898-918, 2019.

[10] R. Miculescu and A. Mihail, "New fixed point theorems for set-valued contractions in b-metric spaces," Journal of Fixed Point Theory and Applications, vol. 19, no. 3, pp. 2153-2163, 2017.

[11] M. Cosentino, M. Jleli, B. Samet, and C. Vetro, "Solvability of integrodifferential problems via fixed point theory in $b$-metric spaces," Fixed Point Theory and Applications, vol. 2015, Article ID 1, 2015.

[12] H. Aydi, M. Abbas, and C. Vetro, "Partial Hausdorff metric and Nadler's fixed point theorem on partial metric spaces," Topology and Its Applications, vol. 159, no. 14, pp. 3234-3242, 2012.

[13] K. Javed, F. Uddin, H. Aydi, A. Mukheimer, and M. Arshad, "Ordered-theoretic fixed point results in fuzzy $b$-metric spaces with an application," Journal of Mathematics, vol. 2021, Article ID 6663707, 2021.

[14] S. Czerwik, "Contraction mappings in b-metric spaces," Acta Mathematica et Informmatica Universitatis Ostraviensis, vol. 1, no. 1, pp. 5-11, 1993.

[15] N. Hussain, D. Dorić, and S. Radenović, "Suzuki-type fixed point results in metric type spaces," Fixed Point Theory and Applications, vol. 126, 2012.

[16] S. G. Matthews, "Partial metric topology," Annals of the New York Academy of Sciences, vol. 728, no. 1, pp. 183-197, 1994.

[17] D. Wardowski, "Fixed points of a new type of contractive mappings in complete metric spaces," Fixed Point Theory and Applications, vol. 2012, Article ID 94, 2012. 